\title{
Control over band structure and tunneling in Bilayer Graphene induced by velocity engineering
}

\author{
Hosein Cheraghchi and Fatemeh Adinehvand \\ School of Physics, Damghan University, 36716-41167, Damghan, Iran
}

(Dated: July 1, 2021)

\begin{abstract}
The band structure and transport properties of massive Dirac Fermions in bilayer graphene with velocity modulation in space are investigated in presence of the previously created band gap. It is pointed out that the velocity engineering is considered as a factor to control the band gap of symmetry-broken bilayer graphene. The band gap is direct and independent of velocity value if velocity modulated in two layers is set up equally. Otherwise, in the case of interlayer asymmetric velocity, not only the band gap is indirect, but also the electron-hole symmetry fails. This band gap is controllable by the ratio of the velocity modulated in the upper layer to the velocity modulated in the lower layer. In more detail, the shift of momentum from the conduction band edge to the valence band edge can be engineered by the gate bias and velocity ratio. A transfer matrix method is also elaborated to calculate four-band coherent conductance through a velocity barrier possibly subjected to a gate bias. Electronic transport depends on the ratio of velocity modulated inside the barrier to the one for surrounding regions. As a result, a quantum version of total internal reflection is observed for enough thick velocity barriers. Moreover, a transport gap originating from the applied gate bias is engineered by modulating velocity of the carriers in the upper and lower layers.
\end{abstract}

PACS numbers: 72.80.Vp,73.22.Pr,73.23.Ad,73.63.-b

\section{INTRODUCTION}

Charge carriers in monolayer graphene at low energies, near the neutrality point, are described by Dirac fermions with a velocity that is independent of wavelength 1 . This unique property proposes an analogous between Dirac fermions and electromagnetic or mechanical waves in optics and acoustics. Furthermore, this brings several unusual electronic properties such as anomalous integer ${ }^{2}$ and fractiona ${ }^{[3}$ quantum Hall effects, electronic focusing by means of a rectangular potential barrier (Veselago lensing ${ }^{4}$, Klein tunneling ${ }^{566}$ and minimal conductivity ${ }^{7}$. Spatial modulation of wave velocity has been originally studied in optics, acoustics and recently in photonic crystals ${ }^{8}$. The idea can be also applied for Dirac fermion waves by defining a velocity barrier as the region in which the Fermi velocity differs from the one in the surrounding background. In analogous with optics, some optical rules are expected to be valid for massless Dirac fermion waves propagating in monolayer graphene sheets 9 .

There are several ways to engineer the Fermi velocity $\left(v_{F}\right)$ by means of a control over the electron-electron interaction in graphene. Enhancement in the electronelectron interaction induces an increase in the Fermi velocity 10 . Furthermore, an enhancement in $v_{F}$ which is logarithmic in the carrier concentration $n$ has been established in experiments and also described by the renormalization group theory 11 . Modifications of curvature of graphene sheet ${ }^{12}$, periodic potentials ${ }^{13}$ and dielectric screening $14 \mid 15$ are some of propositions for engineering $v_{F}$ via the electron-electron interaction. The $v_{F}$ of graphene is inversely proportional to the dielectric constant of the environment embedding graphene sheet ${ }^{16}$. Structures with velocity modulation in space

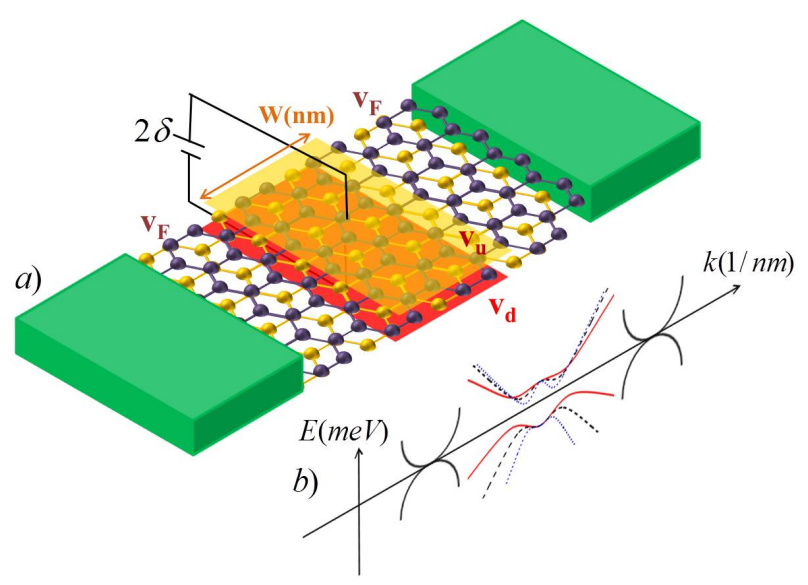

FIG. 1: a) Schematic diagram of bilayer graphene junction in $\mathrm{AB}$ stacking with velocity modulation in space. b) Energy band structure of bilayer graphene for different regions with different velocities. At the same time which vertically gate bias $\delta$ is present, velocity may be experimentally modulated in each layer of BLG. The ratio of velocity modulated in the upper layer $\left(v_{u}\right)$ to the lower layer $\left(v_{d}\right)$ controls the feature of the spectrum as well as tunneling through a velocity barrier.

can be also made by application of appropriate doping 17 or placing a grounded metal plane as a screening plane close to graphene $e^{\sqrt{18}}$. In the presence of the screening planes, speed of carriers is smaller than the speed at isolated graphene sheet. Recently, in a $2 \mathrm{~d}$ electron gas, an artificial graphene has been proposed by modulating a periodic potential of honeycomb symmetry 19 . Electrons in artificial graphene sheets behave like massless Dirac 
fermions with a tunable Fermi velocity.

The electronic properties of monolayer graphene sheets with spatial modulation of the Fermi velocity have been investigated in literature $\sqrt{9|18| 20}-22]$. However, the electronic properties in bilayer graphene (BLG) with an interlayer asymmetric velocity have not been elucidated in detail so far. There are numbers of different experiments in which a controllable direct band gap is observed in gated bilayer graphene $23-26$. However, the amount of current in the off-state still remains high 25|27|29. This off-current has been attributed to several sources 27 such as edge states 30 , the presence of disorder $\frac{31}{31}$, coexistence of massive and massless Dirac fermions in twisted AAstacking bilayer graphene grown on $\mathrm{SiC} 29 \mid 32$. Strain is the other known factor which controls the band gap in $\mathrm{BLG}^{33 \mid 34}$.

In this work, we point out that the velocity modification in symmetry-broken BLG, as an inevitable experimental factor, is able to control the band gap. In the absence of the gate bias $\delta=0$, symmetric or asymmetric velocity modulation in two layers is not able to create a gap in the band structure. However, the previously created gap $\delta \neq 0$ can be controlled by the ratio of modulated velocity in the upper layer to the lower layer $\eta$. The band gap is direct if velocity of itinerant quasi-particles in each layer is set up equally. This gap is independent of velocity, while the momentum attributed to the band gap is inversely proportional to the velocity. On the other hand, the band gap is indirect for non-equal velocities modulated in layers. In this case, the band structure and subsequently the band gap are controlled by $\eta$. The Shift of momentum from the conduction band edge to the valence band edge depends on the gate bias and velocity ratio. Moreover, the electron-hole symmetry fails when $\eta \neq 1$. This kind of control over the band structure which is induced by different velocity modulation in each layer, opens up the possibility of new device applications in nanoelectronics. More importantly, in a BLG under application of gate bias, experiments have to be care about the transition of direct to indirect band gap. This transition can be induced by modification of velocity in layers originating from several experimental requirements such as coating a metallic gate electrode, changing carrier concentration by using application of a gate voltage, strain and etc.

To manifest such a control over the gap, we develop a transfer matrix approach to investigate transport properties through the velocity barrier subjected to a gate bias in BLG. A schematic diagram of the proposed system is presented in Fig, 1 which indicates simultaneous velocity and electrostatic junction. The proposed method is based on a four-band Hamiltonian for AB stacking 35/36. As a result, similar to monolayer graphene ${ }^{9|18| 20}[22$, a total internal reflection occurs for Dirac fermion waves hitting on a thick barrier at the angles of incidence greater than a critical angle. Moreover, it is observed that the transport gap depends on the velocity ratio $\eta$ at large gate bias. This gap is induced by application of a symmetry breaking factor in the barrier region.

We organize this paper as the following: In section II, we present four-band Hamiltonian and a general formula for deriving the spectrum in the presence of velocity modulation in addition to vertically applied gate bias. Then in section III, we switch to calculate transport properties though a velocity junction possibly subjected to an external gate bias in generic form. Finally, the last section includes the results.

\section{HAMILTONIAN AND BAND STRUCTURE IN PRESENCE OF INTERLAYER ASYMMETRY}

The four-band Hamiltonian of bilayer graphene close to the Dirac point (i.e say the valley of $K$ point) for AB stacking is described as the follow:

$$
H=\left(\begin{array}{cc}
-i \hbar v_{u}(\sigma . \nabla)^{\dagger}+V_{u} I & F \\
F & -i \hbar v_{d}(\sigma . \nabla)+V_{d} I
\end{array}\right)
$$

where

$$
F=\left(\begin{array}{cc}
t & 0 \\
0 & 0
\end{array}\right),-i \hbar v \sigma \cdot \nabla=\left(\begin{array}{cc}
0 & \pi^{\dagger} \\
\pi & 0
\end{array}\right)
$$

and $I$ is the unit matrix. Here, $\pi=-i \hbar v\left(\partial_{x}-k_{y}\right)$, $t=390 \mathrm{meV}$ is the coupling energy between the layers. $V_{u}=V_{0}+\delta$ and $V_{d}=V_{0}-\delta$ describe an asymmetric factor which can be applied by a vertically gate bias or doping. This interlayer asymmetry emerges as a difference between on-site energies belonging to each layer. Another interlayer asymmetry can be induced by different modulation in the velocity of itinerant quasi-particles in the upper and lower layers, $v_{u}=\xi_{u} v_{F}$ and $v_{d}=\xi_{d} v_{F}$ respectively. $v_{F}$ is the commonly Fermi velocity used for graphene. $V_{0}$ is the gate voltage applied on both layers setting up to zero. $2 \delta$ is the potential difference between the upper and lower layers induced by a gate bias or doping. The eigen function of the above Hamiltonian 35 is written as $\Psi=\left(\begin{array}{llll}\psi_{A_{2}}^{u} & \psi_{B_{2}}^{u} & \psi_{B_{1}}^{d} & \psi_{A_{1}}^{d}\end{array}\right)^{\top}$. By solving the eigenvalue equation of $H \Psi=E \Psi$, band structure can be calculated in the gapless case or in the presence of previously applied gate bias.

At the same time which vertically gate bias is present, velocity may be experimentally modulated in each layer of BLG. In a gaped BLG, we will show that there is possibility for engineering the previously created gap by using a velocity modulation in each layer. In the presence of a gate bias accompanied with an interlayer asymmetry in velocity, the BLG's spectrum can be extracted from the following equation which presents $k(E)$.

$$
\begin{aligned}
& k(E)^{2}=\left[a \pm \sqrt{a^{2}-b}\right] / v_{u}^{2} \\
& a(E, \eta, \delta)=\left[\eta^{2}(E-\delta)^{2}+(E+\delta)^{2}\right] / 2 \\
& b(E, \eta, \delta)=\eta^{2}\left(E^{2}-\delta^{2}\right)\left(E^{2}-\delta^{2}-t^{2}\right) \\
& \eta=\xi_{u} / \xi_{d}
\end{aligned}
$$




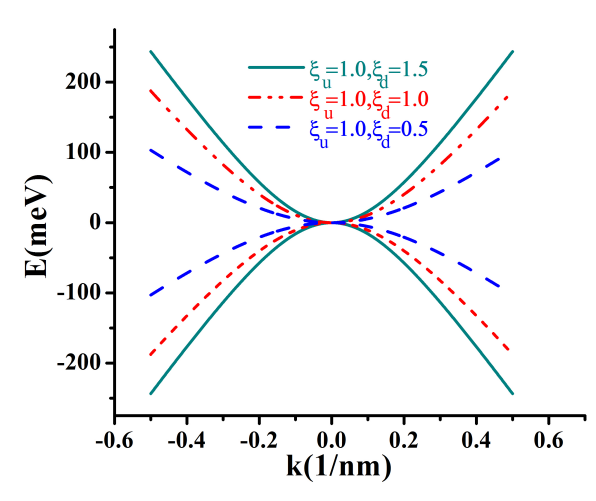

FIG. 2: Bulk band structure of bilayer graphene for several values of velocity modulated in the lower layer $\xi_{d}$ while velocity in the upper layer $\left(\xi_{u}=1\right)$ is fixed. Here, $\xi=v / v_{F}$.

If the gate voltage is nonzero $V_{0} \neq 0$, functions of $a$ and $b$ in the above equation depend on $\varepsilon=E-V_{0}$ instead of $E$. Based on the velocity ratio $(\eta)$ and in the presence of a gate bias $(2 \delta)$, we will indicate that BLG has two different behaviors. For $\eta=1$, BLG behaves as a semiconductor with direct band gap, while for $\eta \neq 1$, it behaves as a semiconductor with indirect band gap. In the case of $\delta=0$, independent of $\eta$, there is no gap in BLG. Bulk band structure calculated by the above equation for $\delta=0$ is shown in Fig.(2) and for $\delta \neq 0$ in Fig.(3) and Fig.(4a).

To investigate the behavior of the energy gap $E_{\text {gap }}$, one can simply derive the following conditions to emerge the extermum points of $E(k)$. Based on Eq. (2), there are two conditions to satisfy the extermum condition $\partial E / \partial k=0$ :

$$
\begin{cases}b=0 & \forall k=0 \\ b=a^{2} & \forall k= \pm v_{u}^{-1} \sqrt{a}\end{cases}
$$

An immediate result from Eq3 3 is that the energy gap $E_{\text {gap }}(\eta)=E_{c}(\eta)-E_{v}(\eta)$ depends on the velocity ratio $\eta$, where $E_{c}$ and $E_{v}$ are the conduction and valence band edges; however, the momentum attributed to the conduction $k_{c}$ and valence $k_{v}$ band edges depend on both variables of $\xi_{u}$ and $\xi_{d}$. The condition $b=0$ results in four eigenvalues of Hamiltonian at the Dirac point calculated as $E= \pm \delta$ and $\pm(t+\delta)$. These eigenvalues and consequently the energy gap appeared at $k=0$ are independent of the velocity ratio $\eta$. The condition $b=a^{2}$ leads to the energy gap at the k-points derived by the following equation: $k_{c / v}\left(\xi_{u}, \xi_{d}\right)= \pm v_{u}^{-1} \sqrt{a\left(E_{c / v}, \eta, \delta\right)}$.

\section{A. Gapless band structure in presence of interlayer symmetric potential}

Let us first concentrate on the gapless case with no external gate biasing $(\delta=0)$ which conserves chiral symmetry. Based on Eq. 22), the four band spectrum for a

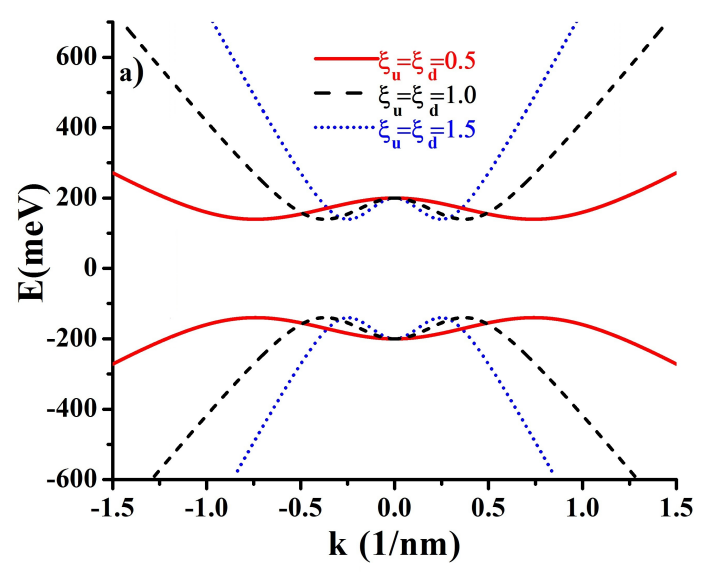

FIG. 3: Bulk band structure of bilayer graphene for the case of the same velocity modulated in the upper and lower layers whenever a band gap is previously created by application of a vertically gate bias. Note that in this case, velocity in two layers are equal to each other but possibly can be different from $v_{F}$. In other word, $\xi=\xi_{u}=\xi_{d}$.

BLG with a tunable velocity in each layer $\left(v_{u} \neq v_{d}\right)$ can be derived as the following 27 ,

$$
\begin{aligned}
& E= \pm \sqrt{\varphi(k)+(-1)^{\epsilon} \sqrt{\varphi^{2}(k)-v_{u}^{2} v_{d}^{2} k^{4}}} . \\
& \varphi(k)=\left(\left(v_{u}^{2}+v_{d}^{2}\right) k^{2}+t^{2}\right) / 2
\end{aligned}
$$

where $\epsilon=1$ and 2 are attributed to the low and high energy bands, respectively. In the case of $\epsilon=1$, there is no band gap at the Fermi Dirac point $(k=0)$. The whole spectrum is robust against the exchange of $v_{u}$ by $v_{d}$. This robustness can also be derived by exchanging of $\eta \rightarrow 1 / \eta$ in Eq.(2). In this case, the only real solutions for the extermum points derived by Eq. (3), are $E=0$ and $\pm t$ which emerge at $k=0$.

The chiral symmetry is conserved even though quasiparticles have different velocities in each layer. In this case, modulation of velocities in each layer just changes the effective mass of quasi-particles. Fig. 22 shows the energy bands of BLG with different velocities in each layer. The band structure is symmetric and behaves as a parabolic form. As a conclusion, without any application of potential difference, only interlayer asymmetry in velocity is not able to break the electron-hole symmetry.

\section{B. Band structure in presence of interlayer asymmetry in potential but symmetry in velocity: Direct band gap}

In the presence of an interlayer asymmetric factor such as an external gate bias $(\delta \neq 0)$ and in the special case of the same velocities setting up on each layer $v_{u}=v_{d}=v$ $(\eta=1)$, the four band spectrum is described as 27 : 
$E^{2}=(v k)^{2}+\delta^{2}+t^{2} / 2+(-1)^{\epsilon} \sqrt{(v k)^{2}\left(4 \delta^{2}+t^{2}\right)+t^{4} / 4+k^{2}}$

As shown in Fig. (3), the low energy band $\epsilon=1$ displays a Mexican hat shape. Despite turning external gate bias on, the band structure still remains symmetric giving rise the electron-hole symmetry. The functions of $a(E, \delta), b(E, \delta)$ defined in Eq. 2) are independent of $\eta$. Therefore, the band gap is independent of the velocity which is modulated in layers. The requisite condition for deriving the band gap $\left(b=a^{2}\right.$ in Eq. (3) ) results in a symmetric solution for the conduction and valence band edges,

$$
E_{c}=-E_{v}=\frac{t \delta}{\sqrt{4 \delta^{2}+t^{2}}} \quad \forall \quad k \neq 0
$$

So the band gap is written as $E_{g a p}=2 E_{c}$. At $k=0$, the gap is fixed to the value $2 \delta$. Because $a\left(E_{v}\right)=a\left(E_{c}\right)$, one can conclude that the momentum of the conduction and valence band edges emerge at the same point $k_{c}=$ $k_{v}=k_{\text {gap }}$ from the center of valley.

$$
k_{g a p}= \pm \frac{2 \delta}{v_{F}} \sqrt{\frac{t^{2}+2 \delta^{2}}{t^{2}+4 \delta^{2}}} \frac{1}{\xi}
$$

Consequently, the band gap is direct and the momentum attributed to the gap is inversely proportional to the velocity $\xi$. For the limit of low external gate bias $\delta \ll t$, the band gap tends to the gate bias $E_{\text {gap }} \rightarrow 2 \delta$. However, for large potential differences $\delta \gg t$, the band gap tends to saturate at the interlayer hopping energy $E_{\text {gap }}^{\text {sat. }} \rightarrow t$. For both limits, the momentum attributed to the band gap behaves as $k_{\text {gap }} \propto 2 \delta / v$. For the case of slower velocity $\xi<1$, the effective mass at the conduction and valence band edges is heavier than the effective mass for the one with faster velocity $\xi>1$.

\section{Band structure in presence of interlayer asymmetry in potential and velocity: Indirect band gap}

In this case, interlayer asymmetry is applied on both of electrostatic potential and also velocity of itinerant quasi-particles $\left(v_{u} \neq v_{d}\right)$. In the case of $\eta \neq 1$ and $\delta \neq 0$, there is an asymmetry between the conduction and valence bands of the spectrum giving rise the electron-hole asymmetry 37 . Consequently, the conduction and valence band edges are appeared at asymmetric energy points measuring from the band center $E=0$. As a result, the momentum attributed to the conduction and valence band edges emerges at different points, $k_{c} \neq k_{v}$. Therefore, the band gap is indirect. The shift of momentum from the conduction band edge to the valence band edge $\left(\Delta k=k_{c}-k_{v}\right)$ depends on velocity in each layer. Although the band gap just depends on the velocity ra-
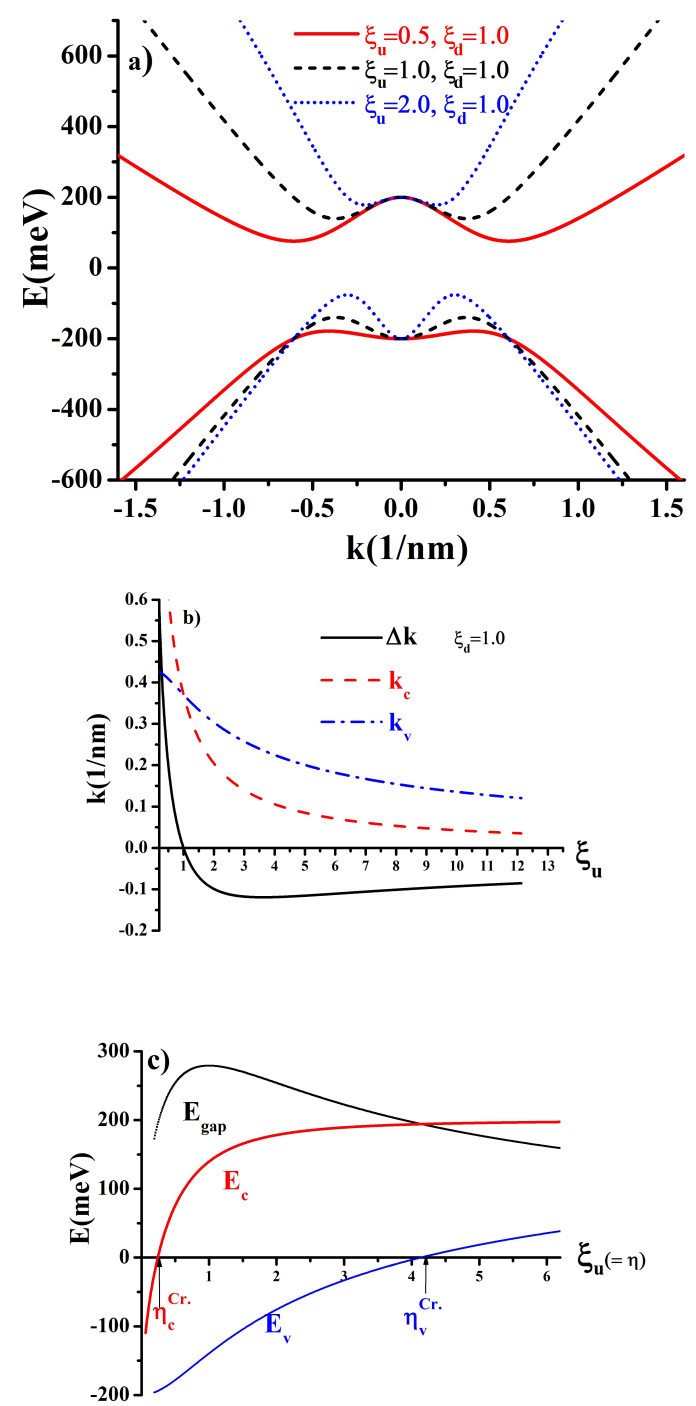

FIG. 4: a) Bulk band structure of bilayer graphene for an interlayer asymmetry in velocity whenever a band gap is previously created by application of a vertically gate bias. Note that velocity modulation is $\xi_{u}$ for the upper layer and fixed for the lower layer $\xi_{d}=1$. For more detail, the electrostatic potential on the upper and lower layers are set $(+\delta)$ and $(-\delta)$, respectively. Here the potential difference is set $2 \delta=400 \mathrm{meV}$. b) The momentum of the conduction $k_{c}$ and valence $k_{v}$ band edges, and also the momentum shift from the conduction band edge to the valence band edge $\Delta k=k_{c}-k_{v}$ as a function of $\xi_{u}$. c) The conduction $E_{c}$ and valence $E_{v}$ band edges and also the energy gap $E_{\text {gap }}$ in terms of $\xi_{u}$ (or here $\eta$ ).

tio $\eta$, however, in a fixed velocity ratio, the whole feature of the spectrum is sensitive to both values of velocity attributed to the upper and lower layers. Let us set velocity of the lower layer to be fixed as $\xi_{d}=1$ while $\xi_{u}$ is tunable. The lower and upper layers are characterized by the electrostatic potential of $-\delta$ and $\delta$, respectively.

The asymmetric band structure is represented in 


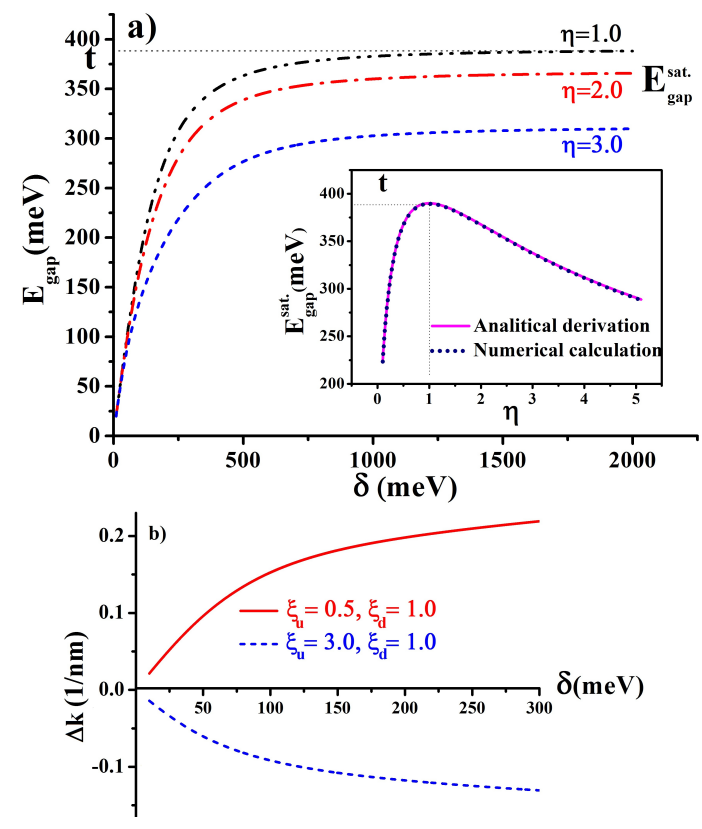

FIG. 5: a) The energy gap in terms of the potential difference between the upper and lower layer for several velocity ratio. The inset figure shows the saturated band gap in terms of the velocity ratio for $\delta=4000 \mathrm{meV}$. The numerical calculation shown in the inset figure confirms the analytical derivation of Eq8. b) The momentum shift from the conduction band edge to the valence band edge in terms of the potential difference between the upper and lower layer for several velocity ratio.

Fig. (44) for three values of velocity of the upper layer $\xi_{u}$. Although the band structure is asymmetric, its form preserves the 'Mexican hat' shape. In the appendix A, we have provided a comparison between the electron-hole asymmetry arising from the full Hamiltonian of BLG and the dominant Hamiltonian which is considered in this work.

Fig. (4 b) shows the momentum attributed to the conduction $k_{c}$ and valence $k_{v}$ band edges and also their momentum shift $\Delta k$ in terms of $\xi_{u}$. As it is obviously observed, both of $k_{c}$ and $k_{v}$ decreases with $\xi_{u}$. Moreover, their curves intersect each other at $\eta=1$ which results in the direct band gap. However, for all values of $\eta \neq 1$, the band gap is indirect. For $\xi_{u}<1$, the momentum shift of $k_{c}$ away from the Dirac point is larger than the momentum shift of $k_{v}$.

By finding roots of Eq. (3), the conduction and valence band edges are computed in terms of system parameters. Fig. (4k) indicates dependence of $E_{c}, E_{v}$ and also $E_{g a p}$ on the velocity ratio $\eta$. The curves related to $E_{c}$ and $E_{v}$ never intersect each other. In all ranges of $\eta, E_{c}>E_{v}$. So BLG always behaves as a semiconductor, not metal nor semi-metal. The energy gap has a maximum at $\eta=1$ in which the gap is direct. A sharp variation of $E_{g a p}$ with $\eta$ is seen for the range of $\eta<1$. Parameters of $\eta_{c}^{c r}$. and $\eta_{v}^{c r}$. are those critical velocity ratios in which $E_{c}$ or $E_{v}$ cross the band center $E=0$. The curvature width of function $E_{g a p}(\eta)$ is measured by $\Delta \eta^{c r .}=\eta_{v}^{c r .}-\eta_{c}^{c r .}$. The critical velocity ratio for the valence and conduction band edges is derived as the following form: $\eta_{v / c}^{c r}=1+2(t / \delta)^{2}[1 \pm$ $\left.\sqrt{1+(\delta / t)^{2}}\right]$. In both limits of $\delta \ll t$ and $\delta \gg t$, the width of the peak which emerges in $E_{g a p}(\eta)$, tends to $\Delta \eta^{c r .} \rightarrow 2 t / \delta$. As a conclusion, for large gate bias $\delta$, there is a sharp variation in the energy gap as a function of the velocity ratio. In large velocity ratio $\eta \rightarrow \infty$, the asymptotic solution of Eq. (3) for the conduction band edge is $E_{c} \rightarrow \delta$. In this limit, the momentum attributed to the conduction band edge behaves as a power law with $v_{u} ; k_{c} \rightarrow 2 \delta / v_{u}$. In the opposite limit of $\eta \rightarrow 0$, the asymptotic solution for the valence band edge is $E_{v} \rightarrow$ $-\delta$. So, the momentum attributed to the valence band edge tends to the constant; $k_{v} \rightarrow 2 \delta / v_{F}$.

Although the energy gap increases with the external gate bias, as it is shown in Fig.(5a), the energy gap is controllable by means of the velocity ratio in large $\delta$. In fact, for $\delta \gg t$, the band gap saturates with the gate voltage at the value which is proportional to the interlayer coupling $(t)$. In this limit, by applying the approximation of $\left(\left|E^{2}-\delta^{2}\right| \gg t^{2}\right)$ in Eq3 3 one can analytically derive that the saturated band gap at $\delta \gg t$ behaves with the velocity ratio as the following form;

$$
E_{\text {gat }}^{\text {sat. }}(\eta)=\frac{2 \sqrt{\eta}}{\eta+1} t
$$

In the special case of $\eta=1$, the band gap saturates at $E_{\text {gaip }}^{\text {sat. }}(\eta=1) \rightarrow t$. As shown in the inset Fig. (5), numerical calculations completely confirm this analytical derivation. The momentum shift $\Delta k$, which measures how much the gap is indirect, can be manipulated by using the gate bias. Fig.(5b) represents the momentum shift from $k_{c}$ to $k_{v}$ in respect of the gate bias for several values of $\xi_{u}$. This momentum shift from $k_{c}$ to $k_{v}$ increases with the gate bias. If we transform the velocity ratio as $\eta \rightarrow 1 / \eta$, in the spectrum feature, the conduction band will be exchanged with the valence band. Furthermore, based on Eq.(3), the band gap is robust against transformation of $\eta \rightarrow 1 / \eta$.

In addition to the direct measurements of the spectrum, the dependence of the energy gap on the velocity ratio can be manifested in transport properties through a velocity junction.

\section{TRANSPORT PROPERTIES ACROSS NON-UNIFORM POTENTIAL AND VELOCITY JUNCTIONS}

Let us consider a BLG sheet in which the velocity of itinerant quasi-particles in the upper and lower layers varies in space; representing as $v_{u}(\vec{r})$ and $v_{d}(\vec{r})$. We assume that variation of velocity is smooth on the scale of the lattice constant. In this section, we outline the approach used to investigate transport properties through a barrier of velocity and potential. 


\section{A. Current Density Operator}

First, by using the continuity equation, we derive the current density operator. The continuity equation is as the following,

$$
\nabla \cdot j=-\partial_{t} \rho
$$

where $\rho=\Psi^{\dagger} \Psi$ is the charge and $j$ is the current density operator. By using the Schroedinger equation, divergence of the current density operator is written as

$$
\nabla \cdot j=\left[(H \Psi)^{\dagger} \Psi-\Psi^{\dagger}(H \Psi)\right] / i \hbar
$$

By substitution of $H$ from Eq 1 and two component spinor as $\Psi=\left(\begin{array}{l}\psi_{u} \\ \psi_{d}\end{array}\right)$ in the above equation, we have

$$
\begin{aligned}
i \hbar \nabla . j= & -\left(\begin{array}{c}
\left(-i \hbar v_{u}(\sigma . \nabla)^{\dagger}+\delta\right) \psi_{u}+F \psi_{d} \\
F \psi_{u}+\left(-i \hbar v_{d}(\sigma . \nabla)-\delta\right) \psi_{d}
\end{array}\right)^{\dagger}\left(\begin{array}{c}
\psi_{u} \\
\psi_{d}
\end{array}\right) \\
& +\left(\begin{array}{ll}
\psi_{u}^{\dagger} & \psi_{d}^{\dagger}
\end{array}\right)\left(\begin{array}{c}
\left(-i \hbar v_{u}(\sigma . \nabla)^{\dagger}+\delta\right) \psi_{u}+F \psi_{d} \\
F \psi_{u}+\left(-i \hbar v_{d}(\sigma . \nabla)-\delta\right) \psi_{d}
\end{array}\right)
\end{aligned}
$$

After simplification, it is derived that interestingly, the current density operator is independent of the gate bias $\delta$ and also the hopping matrix $F$.

$$
\nabla \cdot j=\left[v_{u} \nabla \cdot\left(\psi_{u}^{\dagger} \sigma^{\dagger} \psi_{u}\right)+v_{d} \nabla \cdot\left(\psi_{d}^{\dagger} \sigma \psi_{d}\right)\right]
$$

Therefore, current density operator for a BLG sheet is presented as,

$$
j=\left(\begin{array}{c}
\psi_{u} \\
\psi_{d}
\end{array}\right)^{\dagger}\left(\begin{array}{cc}
v_{u} \sigma^{\dagger} & 0 \\
0 & v_{d} \sigma
\end{array}\right)\left(\begin{array}{l}
\psi_{u} \\
\psi_{d}
\end{array}\right)
$$

Finally, the current density in the i'th region can be written in the following compact form.

$$
j_{i}=\Phi_{i}^{\dagger} \Sigma \Phi_{i}
$$

where the auxiliary spinor is defined as $\Phi_{i}=\widetilde{v}_{i} \Psi_{i}$ and

$$
\Sigma=\left(\begin{array}{cc}
\sigma^{\dagger} & 0 \\
0 & \sigma
\end{array}\right), \quad \widetilde{v}_{i}=\left(\begin{array}{cc}
\sqrt{v_{u}^{i}} & 0 \\
0 & \sqrt{v_{d}^{i}}
\end{array}\right)
$$

\section{B. Transfer Matrix Method}

We assume a plane wave solution for the four-band Hamiltonian. So the wave function in each region with a constant potential is written as the following matrix product, $\Psi(x)=P(x) * A$, where $P(x)$ and $A$ are the plane wave and coefficient matrices, respectively. Detail of matrices $P$ and $A$ are accessible in appendix $B$ and also Refs.(35/38). The local current density in terms of matrices $P(x)$ and $A$ in each region reads as the following form,

$$
j_{i}=A_{i}^{\dagger} P_{i}^{\dagger} \widetilde{v}_{i}^{\dagger} \Sigma \widetilde{v}_{i} P_{i} A_{i}
$$

where the auxiliary spinor in Eq 14 has been replaced by $\Phi_{i}=\widetilde{v}_{i} P_{i} A_{i}$. The continuity equation of $\vec{\nabla} \cdot \vec{j}(\vec{r})=0$ leads to the boundary matching condition at interfaces of a junction. On the other word, conservation of the current density results in the continuity of the auxiliary spinor $\Phi_{i}$ on the boundaries of the barrier junction.

$$
\Phi_{1}=\Phi_{2} \Longrightarrow \widetilde{v}_{2} \Psi_{2}=\widetilde{v}_{1} \Psi_{1}
$$

Referring to the schematic cartoon shown in Fig 1 we consider a simultaneous barrier of velocity,

$$
v(x)=\left\{\begin{array}{cc}
v_{u}=v_{d}=v_{F} & \mathbf{I}: \mathbf{x}<0, \mathbf{I I I}: \mathbf{x}>\mathrm{w} \\
v_{u}, v_{d} & \mathbf{I I}: 0<\mathrm{x}<\mathrm{w}
\end{array}\right.
$$

and electrostatic potential. At the same time, the barrier can be subjected to a gate bias.

$$
V(x)=\left\{\begin{array}{c}
V_{u}=V_{d}=V_{0} \quad \mathbf{I}: \mathbf{x}<0, \mathbf{I I I}: \mathbf{x}>\mathrm{w} \\
\left\{\begin{array}{l}
V_{u}=V_{0}^{\prime}+\delta / 2 \\
V_{d}=V_{0}^{\prime}-\delta / 2
\end{array} \quad \mathbf{I I}: 0<\mathrm{x}<\mathrm{w}\right.
\end{array}\right.
$$

By applying continuity of the auxiliary spinor on the boundaries of the barrier, one can connect the coefficient matrix related to the last region $A_{3}$ to the coefficient matrix for the first region $A_{1}$.

$$
\begin{gathered}
A_{1}=M A_{3} \\
M=P_{1}^{-1}(0) \tilde{v}_{1}^{-1} \tilde{v}_{2} P_{2}(0) P_{2}^{-1}(w) \tilde{v}_{2}^{-1} \tilde{v}_{3} P_{3}(w)
\end{gathered}
$$

where $M$ is the transfer matrix. We assume that the energy range of incidence particles in the first region is limited to the range of $0<\varepsilon_{1}<t 3$. Consequently, the wave numbers $\alpha_{+}^{(1)}$ and $\alpha_{+}^{(3)}$ which are defined in the appendix, $\mathrm{B}$, are real while $\alpha_{-}^{(1)}$ and $\alpha_{-}^{(3)}$ are imaginary. In this range of energy, coefficient matrices in the first and third regions are proposed as the following form.

$$
A_{1}=\left(\begin{array}{llll}
1 & r & 0 & e_{g}
\end{array}\right)^{\top}, A_{3}=\left(\begin{array}{llll}
t & 0 & e_{d} & 0
\end{array}\right)^{\top}
$$

For the first region, $e_{g}$ is the coefficient of growing evanescent state and $r$ is the coefficient of reflection. In the last region, $t$ is the transmission coefficient and $e_{d}$ is the coefficient of decaying evanescent state. By rearrangement of Eq. 18, the coefficient of transmission is derived as a function of the transfer matrix elements as the following;

$$
t=\left[M_{11}-M_{13} M_{31} / M_{33}\right]^{-1} .
$$


The transmission probability of particles through a barrier is defined as the ratio of out-flowing current to inflowing current.

$$
T=\frac{J_{o u t}^{3}}{J_{\text {in }}^{1}}
$$

where $J_{\text {out }}^{3}$ is the out-flowing current in the last region and $J_{i n}^{1}$ is the in-flowing current incidence from the first region. By using Eq, 15 , the transmission probability can be represented as the following form.

$$
T=\frac{\left(\begin{array}{llll}
t & 0 & 0 & 0
\end{array}\right) P_{3}^{\dagger} \tilde{v}_{3}^{\dagger} \Sigma \tilde{v}_{3} P_{3}\left(\begin{array}{l}
t \\
0 \\
0 \\
0
\end{array}\right)}{\left(\begin{array}{llll}
1 & 0 & 0 & 0
\end{array}\right) P_{1}^{\dagger} \tilde{v}_{1}^{\dagger} \Sigma \tilde{v}_{1} P_{1}\left(\begin{array}{l}
1 \\
0 \\
0 \\
0
\end{array}\right)}
$$

The conductance is calculated by using Landauer formalism in the linear regime. Transport is coherent and is calculated at zero temperature. Conductance is proportional to angularly averaged transmission projected along the current direction.

$$
G=2 G_{0} \int_{0}^{\pi / 2} T(E, \varphi) \cos (\varphi) d \varphi
$$

where $G_{0}=e^{2} m v w / \hbar^{2}$.

\section{Transport across a single velocity barrier}

The behavior of a beam produced by Dirac fermions whenever hit on the barrier region, is similar to the behavior of an optical beam passing through dielectric materials. In the subsequent sections, we will show that a quantum mechanical version of well-known laws in geometrical optics can be also applied on the propagation of Dirac fermions in BLG.

Case i: Let us consider tunneling through a single velocity-induced sharp barrier. For a pure velocity barrier, type of quasi-particles inside and outside of the barrier is the same for all ranges of energy. For normal incidence $\theta_{1}=0$ and in absence of any gate bias, transmission coefficient for a velocity barrier with unity velocity ratio $\eta=1$ inside and outside of the barrier can be analytically calculated as

$$
t=e^{i \alpha_{1} w}\left[\cos \left(\alpha_{2} w\right)-i S \sin \left(\alpha_{2} w\right)\right]^{-1}
$$

where

$$
S=\frac{1}{2}\left(\frac{\omega_{1} \alpha_{2}}{\omega_{2} \alpha_{1}}+\frac{\omega_{2} \alpha_{1}}{\omega_{1} \alpha_{2}}\right)
$$
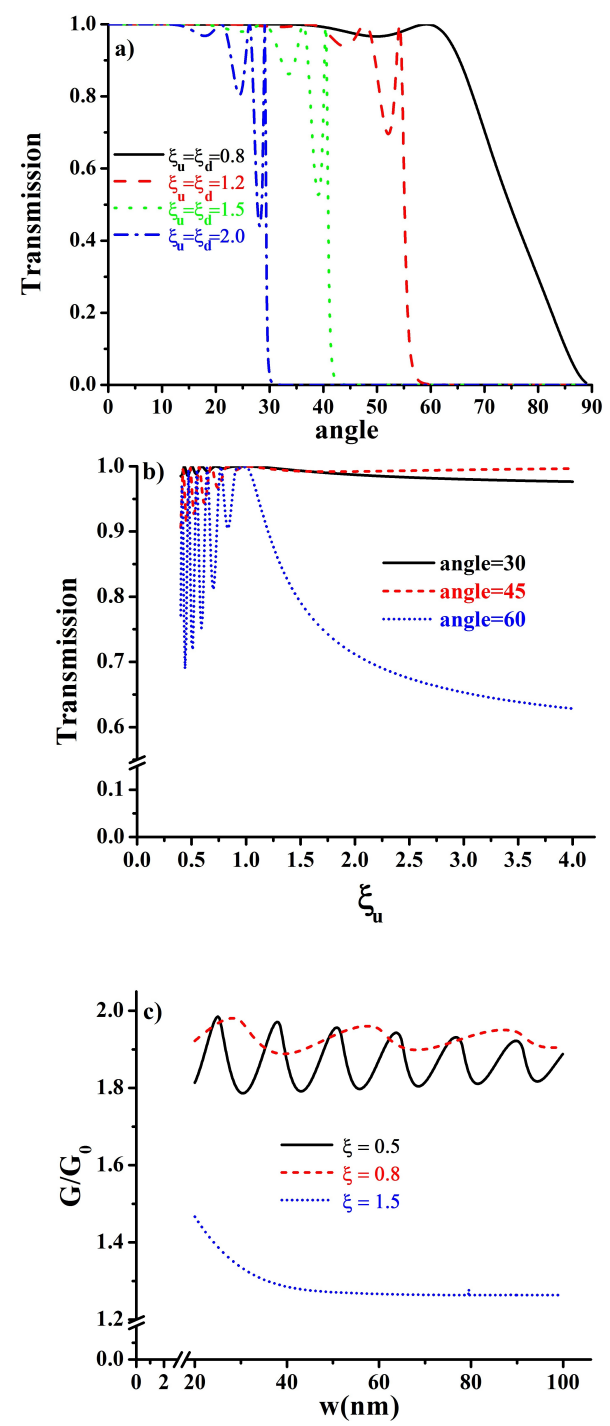

FIG. 6: a) Transmission probability as a function of incidence angle $\theta_{1}$ for the case of the same velocity modulated in both layers. The velocity ratio $\xi_{u}=\xi_{d}=v_{2} / v_{F}$ is set to $0.8,1.2,1.5,2.0$. Estimated critical angle for the velocity ratio 1.2 and 1.5 is about $\theta_{c r} \approx 56$ and 42 . b) Transmission probability in terms of the velocity ratio for several incidence angles. we consider a thick velocity barrier with the width $w=100 \mathrm{~nm}$ for parts (a) and (b). c) Conductance as a function of the width for the velocity ratio equal to $0.5,0.8,1.5$. For all parts, the energy value is set to $E=10 \mathrm{meV}$

and $\alpha_{1}=\left[\omega_{1}^{2}+\omega_{1} t / v_{1}\right]^{1 / 2}, \alpha_{2}=\left[\omega_{2}^{2}+\omega_{2} t / v_{2}\right]^{1 / 2}$ are the wave vectors along the $\mathrm{x}$-axis direction outside and inside the velocity barrier, respectively. Here, scaled energy in each region is defined as $\omega_{1}=E / v_{1}$ and $\omega_{2}=E / v_{2}$. Replacing defined parameters in $S$, results in $S=1$. Therefore, transmission probability is derived as the following form,

$$
T=|t|^{2}=\frac{1}{\left[\cos ^{2}\left(\alpha_{2} w\right)+\sin ^{2}\left(\alpha_{2} w\right)\right]}=1 .
$$


As a result, independence of all barrier parameters, transmission at the normal incidence is always perfect. This behavior is similar to what we expect from the standard Klein tunneling. This transparency at the normal incidence will be demonstrated numerically in Fig 6. At arbitrary incidence angle, the wave vectors along the $\mathrm{x}$ axis direction in the regions I and II can be represented as the following.

$$
\alpha_{1}=\sqrt{\frac{1}{v_{1}^{2}}\left(E^{2}+t E\right)-k_{y}^{2}}, \quad \alpha_{2}=\sqrt{\frac{1}{v_{2}^{2}}\left(E^{2}+t E\right)-k_{y}^{2}}
$$

Suppose that the velocity outside the barrier $v_{1}$ is set to be $v_{F}$. Conservation of the energy $E$ and the component $k_{y}$ of the wave vector across the barrier leads to the following compact form for the wave vector inside the velocity barrier.

$$
\alpha_{2}=k \sqrt{\frac{1}{\xi^{2}}-\sin ^{2} \theta_{1}}
$$

where $\xi=\xi_{u}=\xi_{d}=v_{2} / v_{F}$ is the velocity of quasiparticles inside the barrier scaled by $v_{F}$. $\theta_{1}$ is the incidence angle of quasi-particles which hit on the barrier from the region I. For the range of $\xi>1$, a look at Eq 26 obviously demonstrates that there are some evanecsent modes in the barrier region (in which $\alpha_{2}$ is imaginary) if only the incidence angle $\theta_{1}$ is greater than a critical angle which is defined as,

$$
\theta_{c r .}=\arcsin (1 / \xi)
$$

In analogous with optics, the total internal reflection (TIR) emerges when a Dirac fermion wave hits from a denser medium (region I) on a rarer medium (the barrier region II). This behavior is interpreted as $\xi>1$ in our studied system $\frac{918|20| 22}{2}$. To demonstrate such a critical angle in BLG, we plot transmission probability $T\left(\theta_{1}\right)$ as a function of the incidence angle in Fig 6 for several values of velocity. For $\xi>1$ and $\theta_{1}>\theta_{c r}$. transmission is negligible for enough thick barriers. We have also checked that variation of transmission around the critical TIR angle is more sharp for the multiple structure of velocity barriers in compared with the single velocity barrier. Furthermore, as indicated in Fig.(6b), transmission probability shows a sharp change in behavior at $\xi=1$. In the case of $\eta \neq 1$, the larger velocity modulated in the upper or lower layer, the smaller critical angle emerges. The critical angle just depends on $\xi$. So this property is more appropriate for designing a waveguide based on the BLG substrates 20 .

As a conclusion for $\mathrm{Eq} 26$, for the range of $\xi<1$, the wave vector inside the barrier $\alpha_{2}$ is real which gives rise the propagating modes. Consequently, some resonance states are expected to emerge. The resonance states obey the following resonance condition, $\alpha_{2}\left(\theta_{1}, \xi, E\right) w=n \pi$, where $n$ is the resonant order. As seen in Fig. (6a,b), the
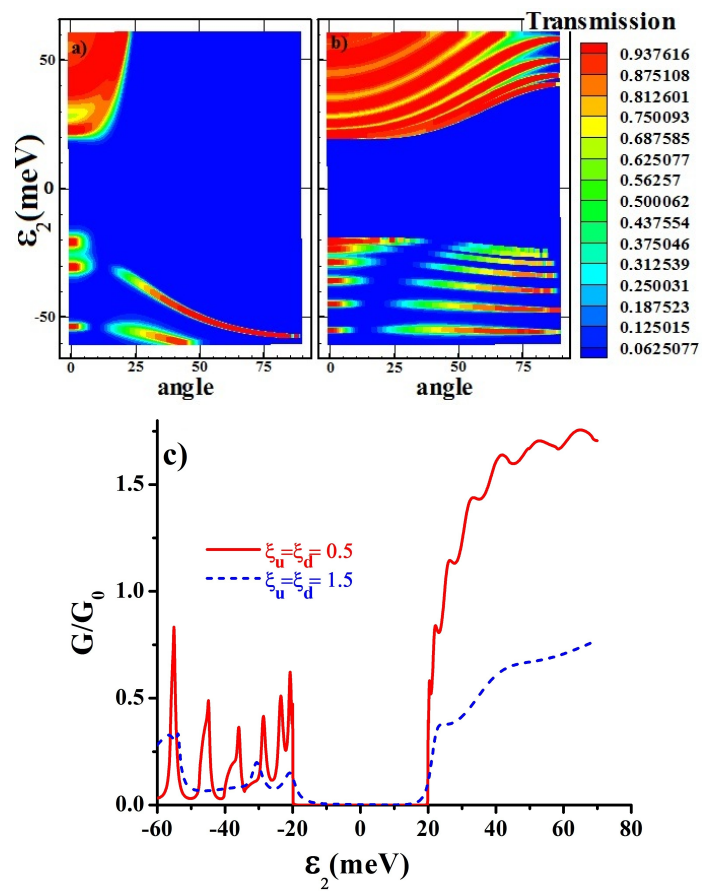

FIG. 7: a) A 3D contour-plot of transmission probability as a function of incidence angle $\theta_{1}$ and energy $\varepsilon_{2}=E-V_{0}^{\prime}$ for the velocity ratio a) $\xi_{u}=\xi_{d}=1.5>1$ and b) $\xi_{u}=\xi_{d}=0.5<1$. c) Conductance in terms of Fermi energy for the velocity ratio $\xi>1$ and $\xi<1$. The gate bias is set to $\delta=40 \mathrm{meV}$. The gate potential applying on both layers is set to $V_{0}^{\prime}=80 \mathrm{meV}$ for inside the barrier and $V_{0}=0$ for outside the barrier. The barrier width is $w=50 \mathrm{~nm}$.

velocity barrier is transparent against the propagation of Dirac fermionic waves at the resonance states. The resonance states emerges at the special values of the incidence angle, the barrier width and those velocities belonging to the range of $\xi<1$. To distinguish the propagating from the evanescent modes, we study conductance as a function of the barrier width in Fig.(6) for several values of velocity. For $\xi<1$, conductance has an oscillatory behavior with the barrier width originating from the propagating modes. on the other hand, conductance drops sharply to zero for $\xi>1$ which is a trace of the evanescent modes inside the barrier.

\section{Transport across velocity barrier in presence of a gate bias}

Case ii: In this case, velocity of carriers changes (still $\eta=1$ ) in the barrier region where a perpendicular gate bias is simultaneously applied $(\delta \neq 0)$. To clarify transport properties of the mentioned system, it is worth to look at the wave number inside the barrier. The wave number along the $\mathrm{x}$-axis direction is presented as 


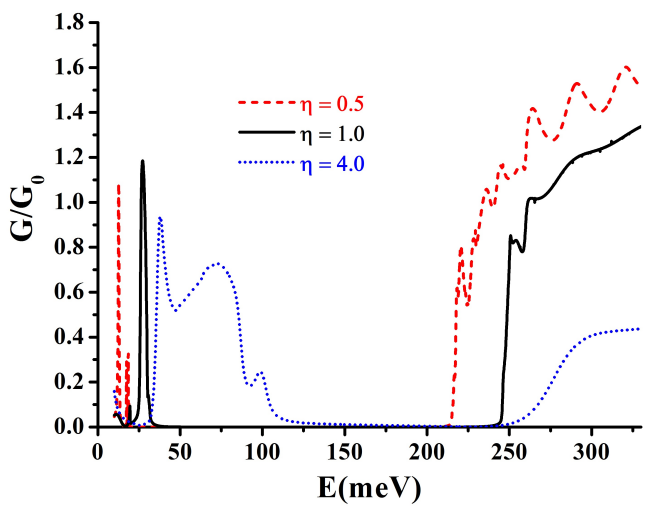

FIG. 8: Conductance in terms of Fermi energy for several values of the velocity ratio $\eta$. Transport gap depends on the velocity ratio. The gate bias is equal to $\delta=125 \mathrm{meV}$. The gate potential applied on both layers is $V_{0}^{\prime}=140 \mathrm{meV}$ for inside the barrier and $V_{0}=0$ for outside the barrier. The barrier width is $w=30 \mathrm{~nm}$.

$$
\alpha_{2}=k \sqrt{\frac{\mu}{\lambda \xi^{2}}-\sin ^{2} \theta_{1}}
$$

where

$$
\begin{gathered}
\mu=\varepsilon_{2}^{2}+\delta^{2}+\sqrt{4 \varepsilon_{2}^{2} \delta^{2}-t^{2}\left(\delta^{2}-\varepsilon_{2}^{2}\right)} \\
\lambda=\varepsilon_{1}^{2}+t \varepsilon_{1}
\end{gathered}
$$

Following procedure of the previous section gives a critical incidence angle as $\theta_{c r .}(E)=\arcsin \left(\sqrt{\frac{\mu}{\lambda}} \frac{1}{\xi}\right)$. Note that in this formula, the critical angle depends on the Fermi energy. Therefore, in the presence of electrostatic gate potentials, this set-up is not proposed as an appropriate material for designing waveguide. However, a definition of the critical angle for such system is useful to interpret the behavior of transmission.

A $3 D$ contour plot of transmission in terms of the incidence angle and energy is indicated in Fig 7 for two velocity values: a) $\xi=1.5>1$ and b) $\xi=0.5<1$. For $\xi=1.5$ and for energies greater than the gap $\varepsilon_{2}>0$, the existence of a critical angle is clearly demonstrated in Fig. (7a) which shows sharp dropping of transmission to zero. However, such a sharp critical angle is absent for $\xi=0.5$. As a conclusion, in addition to the parameters $\mu$ and $\lambda$ which are energy dependence, the velocity $\xi$ still can play an important role to tune transporting modes. To manifest such a property, we study conductance as a function of energy in Fig.(7) for $\xi=0.5$ and 1.5. It is interesting that conductance for the velocity $\xi=0.5$, is much greater than its value for $\xi=1.5$. Moreover, conductance has an oscillatory behavior with the Fermi energy if $\xi<1$. However, it behaves smoothly with the energy if $\xi>1$.
Case iii: In the last case, in addition to the gate biasing $(\delta \neq 0)$, we modulate velocity in layers not to be equal to each other $\eta \neq 1$. Since the band gap of the barrier portion depends on the velocity ratio $\eta$, we expect to manifest this property by concentrating on the transport gap. Fig 8 represents conductance in terms of energy for several values of the velocity ratio $\eta$. What is novel is that the transport gap appeared in conductance depends on the velocity ratio $\eta$. The behavior of the conduction and valence band edges with the velocity ratio is in good agreement with those shown in Fig.(44).

Referring to Fig. (5a), dependence of the band gap on the velocity ratio is strong when the gate bias is large. So one can observe that the transport gap depends on the velocity ratio. The transport gap is remarkable when a thick velocity barrier is manipulated in the presence of a large gate bias $\delta$. Maximum band gap emerges at $\eta=1$.

\section{CONCLUSION}

In the presence of a previously applied gate bias, the electronic band structure of bilayer graphene is investigated when quasi-particles have different Fermi velocity in each layer. We address that the velocity engineering is one of the inevitable experimental factors which affects the transport gap in the broken-symmetry BLG.

In absence of any electrostatic potential, only the modulation of velocity in layers does not cause to open a band gap. In other words, the chiral symmetry conserves for purely velocity modulation $\delta=0$ while this symmetry will break when a gate bias is subsequently applied on BLG. It should be noted that in the presence of a gate bias $\delta \neq 0$, the electron-hole symmetry preserves whenever the same velocity is modulated in both layers; $\eta=1$. In addition, the band structure keeps its 'Mexican hat' shape with a direct band gap. Moreover, the band gap is independent of velocity value. The maximum value of the band gap occurs at $\eta=1$. The momentum attributed to the band gap is inversely proportional to the velocity.

In a generic case, non-equal velocities in two layers $(\eta \neq 1)$ result in the transition of the direct-to-indirect band gap. The band gap depends on the velocity ratio $\eta$ and has a peak at $\eta=1$. Interestingly, the electron-hole symmetry fails, however the band structure still keeps its 'Mexican hat' shape. The shift of momentum from the conduction band edge to the valence band edge is increased with the gate bias.

In the second part, we elaborate a transfer matrix method to calculate coherent tunneling through a velocity barrier possibly subjected to a gate potential. In analogous with optics, we propose a total internal reflection angle $\theta_{c r}$. so that transmission becomes sharply negligible for the incidence angles larger than $\theta_{c r}$. The transport gap which is induced by application of the gate bias in the barrier region, depends on the velocity ratio. 


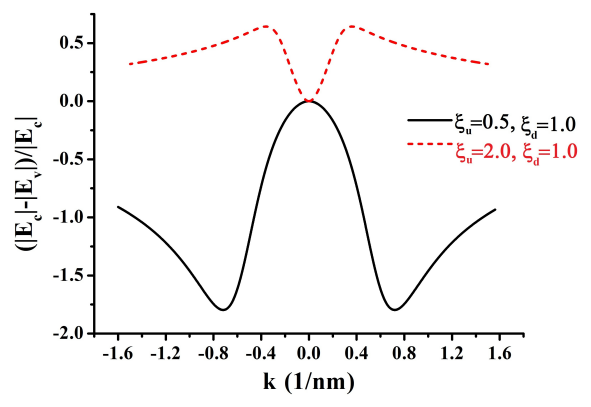

FIG. 9: The electron-hole asymmetric factor as a function of momentum. The gate bias is equal to $\delta=400 \mathrm{meV}$.

\section{ACKNOWLEDGEMENT}

We highly acknowledge R. Asgari for his useful comments during improvement of this work. One of the authors, (H.C), thanks the institute for research in fundamental sciences (IPM) and also the international center for theoretical physics (ICTP) for their hospitality and support during a visit in which part of this work was done. We should also thank M. Barbier for his comments in the four-band tunneling.

\section{Appendix A: The electron-hole asymmetry}

To measure the electron-hole (e-h) asymmetry, we define the e-h asymmetric factor as the following; $\left(\left|E_{c}\right|-\mid\right.$ $\left.E_{v} \mid\right) /\left|E_{c}\right|$. For the case of equal velocities modulated in both layers $\eta=1$, the e-h asymmetric factor is zero for the studied Hamiltonian shown in Eq1. However, as seen in Fig 9, this asymmetric factor increases with the momentum very faster than a linear behavior ${ }^{37}$. This factor reaches to the value of 2 in the special momentum. It is interesting that by application of the transformation of $\eta \rightarrow 1 / \eta$, the e-h asymmetric factor behaves as $r \rightarrow 1 / r$.

In addition to the velocity modulation, the e-h asymmetry is also originated from the inter-layer coupling $\left(\gamma_{4}\right)$ between $A 1-A 2$ and $B 1-B 2$ sites $27 / 37$. At the first order approximation, we have not considered such term in the dominant Hamiltonian shown in Eq[1. In fact, the most important terms which affect the main feature of the band structure are $\gamma_{0}$ and $\gamma_{1}=t$. Here, $\gamma_{0}$ is the intralayer hopping between $A 1-B 1$ and $A 2-B 2$ sites which is proportional to $v_{F}$ in the tight-binding approximation and $\gamma_{1}=t$ is the inter-layer coupling between $A 2-B 1$ sites. The e-h asymmetric factor caused by parameter $\gamma_{4}$, behaves as $4 \gamma_{4} / \gamma_{0} \sqrt{27}$. The well-established values 27 for the hopping parameters are equal to $\gamma_{4} \approx 0.15 \mathrm{eV}$ and $\gamma_{0} \approx 3 \mathrm{eV}$. So the e-h asymmetric factor originating from $\gamma_{4}$ is in order of magnitude $0.1^{27 / 37}$. As a conclusion, in the presence of the previously created band gap, the e-h asymmetry arising from the velocity engineering is a dominant factor in compared with the e-h asymmetry caused by parameter $\gamma_{4}$.

\section{Appendix B: Wave Function}

The eigenfunction of four band Hamiltonian of Eq1 is defined with the following spinor.

$$
\Psi(x)=P(x) A
$$

where coefficient matrix is written as

$$
A=\left(\begin{array}{llll}
U_{A_{2}} & U_{B_{2}} & D_{B_{1}} & D_{A_{1}}
\end{array}\right)^{\top}
$$

and plane wave matrix is presented as $35 \mid 38$

$$
P(x)=\left(\begin{array}{cccc}
e^{i \alpha_{+} x} & e^{-i \alpha_{+} x} & e^{i \alpha_{-} x} & e^{-i \alpha_{-} x} \\
f_{+}^{+} e^{i \alpha_{+} x} & f_{+}^{-} e^{-i \alpha_{+} x} & f_{-}^{+} e^{i \alpha_{-} x} & f_{-}^{-} e^{-i \alpha_{-} x} \\
s_{+} e^{i \alpha_{+} x} & s_{+} e^{-i \alpha_{+} x} & s_{-} e^{i \alpha_{-} x} & s_{-} e^{-i \alpha_{-} x} \\
g_{+}^{+} s_{+} e^{i \alpha_{+} x} & g_{+}^{-} s_{+} e^{-i \alpha_{+} x} & g_{-}^{+} s_{-} e^{i \alpha_{-} x} & g_{-}^{-} s_{-} e^{-i \alpha_{-} x}
\end{array}\right)
$$

$$
\begin{gathered}
f_{+}^{ \pm}=v_{u} \frac{ \pm \alpha_{+}-i k_{y}}{\varepsilon-\delta}, \quad f_{-}^{ \pm}=v_{u} \frac{ \pm \alpha_{-}-i k_{y}}{\varepsilon-\delta} \\
g_{+}^{ \pm}=v_{d} \frac{ \pm \alpha_{+}+i k_{y}}{\varepsilon+\delta} \quad g_{-}^{ \pm}=v_{d} \frac{ \pm \alpha_{-}+i k_{y}}{\varepsilon+\delta} \\
s_{ \pm}=\frac{(\varepsilon-\delta)^{2}-v_{u}^{2}\left[\left(\alpha_{ \pm}\right)^{2}+k_{y}^{2}\right]}{t(\varepsilon-\delta)}, \quad \varepsilon_{i}=E-V_{i}
\end{gathered}
$$

where $\alpha_{+}$and $\alpha_{-}$are the wave vectors along the current direction $(x)$ which is defined as

$$
\alpha_{ \pm}=\sqrt{a(\varepsilon, \eta, \delta)-v_{u}^{2} k_{y}^{2} \pm \sqrt{a(\varepsilon, \eta, \delta)^{2}-b(\varepsilon, \eta, \delta)}} / v_{u}
$$

If the gate voltage turns on, $a$ and $b$ defined in Eq2 are function of $\varepsilon$ in stead of $E$.
* Electronic address: cheraghchi@du.ac.ir

1 A. H. Castro Neto, et al., Rev. Mod. Phys. 81, 109 (2009).

2 V. P. Gusynin and S. G. Sharapov, Phys. Rev. Lett. 95, 146801 (2005).

3 X. Du, I. Skachko, F. Duerr, A. Luican, and E. Y. Andrei, Nature 462, 192 (2009); K. I. Bolotin, F. Ghahari, M. D. Shulman, H. L. Stormer, and P. Kim, Nature 462, 196 (2009).
4 V. V. Cheianov, V. Falko, and B. L. Altshuler, Science 315, 1252 (2007).

5 J. R. Williams, L. DiCarlo, and C. M. Marcus, Science 317, 638 (2007).

6 N. Stander, B. Huard, and D. Goldhaber-Gordon, Phys. Rev. Lett. 102, 026807 (2009) .

7 K. S. Novoselov, A. K. Geim, S. V. Morozov, D. Jiang, M. I. Katsnelson, I. V. Grigorieva, S. V. Dubonos, and A. A. 
Firsov, Nature 438, 197 (2005); ibid: Nat. Phys. 2, 177 (2006).

8 J. D. Joannopoulos, et al., Photonic Crystal: Molding the Flow of Light, Princeton University Press, Princeton NJ, 2008.

9 A. Concha and Z. Tesanovic, Phys. Rev. B. 82, 033413 (2010).

10 P. E. Trevisanutto, C. Giorgetti, L. Reining, M. Ladisa, V. Olevano, Phys. Rev. Lett. 101, 226405 (2008).

11 D. C. Elias, et al., Nat. Phys. 7, 701 (2011).

12 X. Du, I. Skachko, et al.,Nat. Nano. 3, 491 (2008).

13 C. H. Park, et al., Nat. Phys. 4, 213 (2008).

14 D. A. Siegel, et al., Natl. Acad. Sci. USA 108, 11365 (2011).

15 C. Jang, et al., Phys. Rev. Lett. 101, 146805 (2008).

16 H. Choongyu, et al., Nat. Scientific Reports 2, 590 (2012).

17 C. Attaccalite and A. Rubio, Phys. Status. Solidi. B 246, 2523 (2009); M. Polini, et al., Solid. State. Commu. 143, 58, (2007); G. Borghi, et al., Solid. State. Commu. 149, 1117, (2009).

18 A. Raoux, M. Polini, et al., Phys. Rev. B 81, 073407 (2010); G. Borghi, et al., solid state commun. 149, 1117 (2009).

19 C. H. Park, S. G. Louie, Nano Lett. 9, 1793 (2009); M. Gibertini, et al., Phys. Rev. B 79, 241406 (2009).

20 J. H. Yuan, et al., J. Appl. Phys. 110, 103706 (2011); JianHui Yuann, et al., Physica B 406, 4214 (2011).

21 P. M. Krstajic, P. Vasilopoulos, J. Phys. Cond. Matt. 23, 135302 (2011).
22 N. M. R. Peres, J. Phys. Cond. Matt. 21,095501 (2009).

23 T. Ohta, et al., Science 313, 951 (2006).

24 E. Castro, et al., Phys. Rev. Lett. 99, 216802 (2007).

25 J. Oostinga, et al., Nature Mater. 7, 151 (2008).

${ }^{26}$ Y. Zhang, et al., Nature, 459, 820 (2009).

27 E. Mccann, M. Koshino, Rep. Prog. Phys. 76, 056503(2013).

28 I. Martin, et al., Nature Phys. 7, 38-42 (2010); R. Weitz, et al., Science 330, 812-816 (2010).

29 P. Hofmann, Nature Mater. 12, 874875 (2013).

30 J. Li, et al., Nature Phys. 738 (2011).

31 E. V. Castro, et al., Phys. Rev. Lett. 104, 036802 (2011); E. Rossi, S. Das Sarma, Phys. Rev. Lett. 107, 155502 (2011).

32 K. S. Kim, et al., Nature Mater. 12, 887892 (2013).

33 S-M. Choi, S-H. Jhi, and Y-W. Son , Nano. Lett. 10, 3486 (2010).

34 B. Verberck, B. Partoens, F. M. Peeters, and B. Trauzettel, Phys. Rev. B. 85, 125403 (2012).

35 M. Barbier, P. Vasilopoulos, F. M. Peeters and J. M. Pereira, Phys. Rev. B. 79, 155402 (2009)

36 B. Van Duppen, F. M. Peeters, Phys. Rev. B. 87, 205427 (2013).

37 M. Mucha-Kruczynski, et al., Semicond. Sci. Technol. 25, 033001(2010).

38 H. cheraghchi, F. Adinehvand, J. Phys. Cond. Matt. 24, 045303 (2012). 